\title{
MEDULOBLASTOMA EM ADULTOS
}

\section{ANÁLISE DE UMA CASUÍSTICA E RESULTADOS CIRÚRGICOS}

\author{
PAULO HENRIQUE AGUIAR*, MARCELO PRUDENTE*, ALEXANDRE BRUNO RAUL FREITAS*, \\ JOSÉ MARCUS ROTTA*, JOSÉ PINDARO PEREIRA PLESE*, MARIO SERGIO DUARTE ANDRIOLI*
}

RESUMO - Analisamos 15 casos de meduloblastoma em adultos ( 8 homens e 7 mulheres), com idade média de 23,7 anos (variação de 13 a 46) submetidos a ressecção cirúrgica no período de fevereiro de 1988 a outubro de 1995. Os tumores se localizavam no hemisfério cerebelar em 7 casos ( 1 com extensão supratentorial e outro atingindo a cisterna do ângulo pontocerebelar), no vermis e hemisférico em 4 e apenas no vermis em outros 4 . A ressecção foi considerada radical em 7 casos, subtotal em 7 e parcial em um. Não houve mortalidade operatória. Aspectos relativos ao comportamento biológico e prognóstico são discutidos.

PALAVRAS-CHAVE: fossa craniana posterior, meduloblastoma, tumor neuroectodérmico primitivo, adulto.

\section{Medulloblastoma in adulthood: analysis of a casuistics and surgical results}

ABSTRACT - We report on 15 cases of medulloblastoma of adult onset ( 8 male and 7 female) operated upon posterior fossa approach from February 1988 to October 1995. Tumors were localized in cerebellar hemisphere in 7 cases (one with extension to supratentorial notch and another case reaching the cerebello-pontine angle cistern), in vermis and hemisphere in four, only in vermis in another four. Resection was total in seven patients, subtotal in other seven, and partial in one.There was no operative mortality. Aspects regarding biological behavior, diagnosis, pathological findings, surgery and survival are discussed as well as prognostic factors.

KEY WORDS: posterior cranial fossa, medulloblastoma, primitive neuroectodermic tumor, adult hood.

Desde 1925, quando Bailey e Cushing ${ }^{1}$ reconheceram e denominaram o meduloblastoma como um processo distinto dos gliomas e sarcomas, ele tem sido reconhecido como um tumor cerebelar maligno relacionado à faixa etária pediátrica ${ }^{2,3}$, com um pico de incidência aos 5 anos de idade $^{4}$. Entretanto o seu aparecimento em adolescentes e adultos não é infreqüente. Em seu artigo original, Bailey e Cushing descreveram 9 pacientes com idades superiores a 18 anos, portadores de meduloblastoma, que possuíam semelhanças em relação ao comportamento clínico e aspecto histopatológico. O comportamento biológico do meduloblastoma do adulto bem como seus aspectos histopatológicos têm sido estudados, procurando-se estabelecer parâmetros para avaliação prognóstica ${ }^{2,4-15}$. O padrão de resposta ao tratamento cirúrgico e radioterápico difere entre o grupo adulto e o pediátrico ${ }^{2,7-9,16}$, com recorrência local mais frequente no primeiro ${ }^{12}$.

O objetivo deste artigo é mostrar nossa experiência cirúrgica com meduloblastomas no adulto e enfatizar os fatores relativos ao comportamento clínico, diagnóstico, cirurgia, bem como a análise de complicações e sobrevida, ressaltando possíveis fatores prognósticos.

*Serviço de Neurocirurgia do Hospital das Clínicas da Faculdade de Medicina da Universidade de São Paulo (FMUSP): Grupo de Tumores Encefálicos e Metástases. Aceite: 14-setembro-1999.

Dr. Paulo Henrique Aguiar - Rua Custódio de Oliveira 280-71 05630-050 São Paulo SP - Brasil. Fax 011282 6822. E-mail: PHA_Neuro@hotmail.com 


\section{MÉTODO}

Foram analisados retrospectivamente 15 prontuários completos, de pacientes com idade superior a 13 anos ( 8 homens e 7 mulheres), portadores de meduloblastoma de cerebelo, operados no Serviço de Neurocirurgia do Hospital das Clínicas da FMUSP, no período de fevereiro de 1988 a outubro de 1995. Dados relativos ao intervalo entre início dos sintomas e diagnóstico, tipo de sintoma e sinais neurológicos, diagnóstico radiológico, cirurgia, complicações, recidiva e tempo de sobrevida foram coletados para análise e discussão (Tabelas 1 e 2)

Tabela 1. Aspectos clínicos.

\begin{tabular}{|c|c|c|c|c|c|c|c|}
\hline Caso & $\begin{array}{l}\text { Idade } \\
\text { (anos) }\end{array}$ & Sexo & $\begin{array}{l}\text { Sintoma } \\
\text { inicial }\end{array}$ & $\begin{array}{c}\text { Exame } \\
\text { neurológico }\end{array}$ & $\begin{array}{l}\text { Intervalo } \\
\text { sintoma e } \\
\text { diagnóstico } \\
\text { (Meses) }\end{array}$ & Localização Hic & Hidrocefalia \\
\hline 1. RJS & 18 & M & $\begin{array}{c}\text { cefaléia + alt. } \\
\text { marcha }\end{array}$ & $\begin{array}{l}\text { Síndrome HIC e } \\
\text { cerebelar global }\end{array}$ & $<1$ & $\begin{array}{l}\text { Vermiano e } \\
\text { Hemisfério D }\end{array}$ & $\operatorname{sim}$ \\
\hline 2. ERM & 14 & $\mathrm{~F}$ & cefaléia & Síndrome HIC & 3 & Hemisfério E & $\operatorname{sim}$ \\
\hline 3. CJS & 14 & M & cefaléia & $\begin{array}{l}\text { Síndrome HIC e } \\
\text { cerebelar global }\end{array}$ & 2 & Vermiano & $\operatorname{sim}$ \\
\hline 4. MS & 15 & $\mathrm{~F}$ & cefaléia & $\begin{array}{c}\text { Síndrome HIC e } \\
\text { cerebelar apendicular }\end{array}$ & 3 & $\begin{array}{l}\text { Hemisfério D com } \\
\text { ext. supratentorial }\end{array}$ & $\operatorname{sim}$ \\
\hline 5. SCS & 35 & $\mathrm{~F}$ & vômitos & $\begin{array}{l}\text { Síndrome HIC e } \\
\text { cerebelar global }\end{array}$ & 12 & $\begin{array}{l}\text { Vermiano com } \\
\text { ext. supratentorial } \\
\text { e hemisfério D }\end{array}$ & $\operatorname{sim}$ \\
\hline 6. SPO & 23 & $\mathrm{~F}$ & diplopia & $\begin{array}{l}\text { Síndrome HIC e } \\
\text { cerebelar apendicular }\end{array}$ & 36 & Hemisfério D & não \\
\hline 7. JRBO & 34 & M & cefaléia & $\begin{array}{l}\text { Síndrome HIC e } \\
\text { estado comatoso }\end{array}$ & 1 & $\begin{array}{l}\text { Hemisfério } \\
\text { cerebelar }\end{array}$ & $\operatorname{sim}$ \\
\hline 8. AOF & 24 & $\mathrm{~F}$ & cefaléia & Síndrome de HIC & 2 & Vermiano e hemisfério $\mathrm{E}$ & E sim \\
\hline 9. CSL & 18 & M & coma & Síndrome de HIC & $<1$ & Vermiano & $\operatorname{sim}$ \\
\hline 10. NMS & 22 & $\mathrm{~F}$ & $\begin{array}{l}\text { hipoacusia } \\
\text { súbita }\end{array}$ & $\begin{array}{l}\text { Síndrome de nervos } \\
\text { cranianos V,VII, VIII } \\
\text { e IX a E e cerebelar } \\
\text { apendicular E }\end{array}$ & 15 & $\begin{array}{l}\text { Hemisfério E } \\
\text { junto ao ângulo } \\
\text { ponto-cerebelar }\end{array}$ & $\operatorname{sim}$ \\
\hline 11. PLS & 32 & M & cefaléia & $\begin{array}{c}\text { Síndrome } \\
\text { cerebelar e HIC }\end{array}$ & $?$ & & $\operatorname{sim}$ \\
\hline 12. $\mathrm{AB}$ & 23 & M & cefaléia & $\begin{array}{l}\text { Síndrome HIC } \\
\text { e cerebelar global }\end{array}$ & 3 & $\begin{array}{l}\text { Vermiano e hemisferio } \\
\text { cerebelar E }\end{array}$ & o $\operatorname{sim}$ \\
\hline 13. ARC & 15 & $\mathrm{~F}$ & $\begin{array}{l}\text { incoordenação } \\
\text { motora }\end{array}$ & $\begin{array}{c}\text { Síndrome } \\
\text { cerebelar global }\end{array}$ & $?$ & Vermiano & $\operatorname{sim}$ \\
\hline 14. GDS & 13 & M & cefaléia & $\begin{array}{l}\text { Síndrome HIC } \\
\text { e cerebelar axial }\end{array}$ & 9 & Vermiano & $\operatorname{sim}$ \\
\hline 15. MJP & 46 & M & cefaléia & $\begin{array}{l}\text { Síndrome HIC e } \\
\text { cerebelar global }\end{array}$ & 2 & Hemisfério D & $\operatorname{sim}$ \\
\hline
\end{tabular}

M, masculino; F, Feminino; D, direita; E, esquerda; HIC, hipertensão intracraniana;alt, alteração; ext, extensão. 
Tabela 2. Aspectos da terapêutica.

\begin{tabular}{|c|c|c|c|c|c|c|c|}
\hline Caso & Cirurgia & Posição & Derivação & $\begin{array}{l}\text { Grau de } \\
\text { ressecção }\end{array}$ & Complicações & $\begin{array}{c}\text { Tratamento } \\
\text { complementar }\end{array}$ & $\begin{array}{l}\text { Seguimento } \\
\text { (Meses) } \\
\text { Karnofsky }\end{array}$ \\
\hline 1. & $\begin{array}{c}\text { Craniectomia } \\
\text { suboccipital } \\
\text { unilateral D }\end{array}$ & $\begin{array}{c}\text { Semi } \\
\text { sentada }\end{array}$ & DVE Periop. & Total & $\begin{array}{c}\text { Hematoma } \\
\text { extradural } \\
\text { fossa posterior }\end{array}$ & RDT neuroeixo & $30 \backslash 100$ \\
\hline 2 & $\begin{array}{l}\text { Craniectomia } \\
\text { suboccipital } \\
\text { unilateral E }\end{array}$ & $\begin{array}{c}\text { Semi } \\
\text { sentada }\end{array}$ & $\begin{array}{l}\text { DVP prévia } \\
\text { (1 semana) }\end{array}$ & Total & $\begin{array}{l}\text { Hematoma leito } \\
\text { cirúrgico nervos } \\
\text { bulbares IX } \backslash \mathrm{X}\end{array}$ & $\begin{array}{l}\text { RDT neuroeixo eQT } \\
\text { pós recidiva }\end{array}$ & $\begin{array}{l}\text { 12\recidiva } \\
\text { hemisfério } \\
\text { D-40 }\end{array}$ \\
\hline 3 & $\begin{array}{c}\text { Craniectomia } \\
\text { suboccipital }\end{array}$ & $\begin{array}{c}\text { Semi } \\
\text { sentada }\end{array}$ & DVE Periop. & Total & Nenhuma & RDT neuroeixo & $7 \backslash 100$ \\
\hline 4 & $\begin{array}{l}\text { Craniectomia } \\
\text { subocipital D }\end{array}$ & $\begin{array}{c}\text { Semi } \\
\text { sentada }\end{array}$ & DVE periop. & Total & Nenhuma & $\begin{array}{l}\text { RDT neuroeixo e QT, } \\
\text { reoperaçâo após } 1^{\circ} \\
\text { recidiva e DVP }\end{array}$ & $\begin{array}{l}28 \backslash 1 \text { recidiva local } \\
\text { (após } 23 \text { meses) } 80, \\
2^{\circ} \text { recidiva em tronco } \\
\text { (após } 5 \text { meses) } 40 \text { :óbito }\end{array}$ \\
\hline 5 & $\begin{array}{l}\text { Craniectomia } \\
\text { suboccipital } \\
\text { bilateral }\end{array}$ & $\begin{array}{c}\text { Semi } \\
\text { sentada }\end{array}$ & $\begin{array}{l}\text { Cateter de } \\
\text { Lapras } \\
\left(\text { III }{ }^{\circ} \mathrm{V}-\right. \\
\text { Subarac) }\end{array}$ & $\begin{array}{l}\text { Subtotal } \\
(90 \%)\end{array}$ & $\begin{array}{l}\text { Hematoma } \\
\text { em leito } \\
\text { cirúrgico }\end{array}$ & $\begin{array}{c}\text { RDT } \\
\text { neuroeixo }\end{array}$ & $47 \backslash 100$ \\
\hline 6 & $\begin{array}{c}\text { Craniectomia } \\
\text { suboccipital D }\end{array}$ & $\begin{array}{c}\text { Semi } \\
\text { sentada }\end{array}$ & Não & Subtotal & Nenhuma & $\begin{array}{l}\text { RDT neuroeixo, } \\
\text { reoperação após } \\
1^{\circ} \text { recidiva e QT } \\
\text { após } 2^{\circ} \text { recidiva }\end{array}$ & $\begin{array}{c}72 \backslash 1^{\circ} \text { recidiva } \\
\text { local (54 meses) } 100, \\
2^{\circ} \text { recidiva (após } 4 \\
\text { meses) } 50\end{array}$ \\
\hline $\begin{array}{l}7 \\
\text { la }\end{array}$ & $\begin{array}{l}\text { Craniectomia } \\
\text { suboccipital bi- } \\
\text { teral e laminec- } \\
\text { tomia de } \mathrm{C} 1\end{array}$ & $\begin{array}{c}\text { Semi } \\
\text { sentada }\end{array}$ & $\begin{array}{c}\text { DVP } \\
\text { (1 semana) }\end{array}$ & Total & Nenhuma & RDT neuroeixo & $5 \backslash 100$ \\
\hline 8 & $\begin{array}{l}\text { Craniectomia } \\
\text { suboccipital } \\
\text { bilateral }\end{array}$ & $\begin{array}{c}\text { Semi } \\
\text { sentada }\end{array}$ & $\begin{array}{c}\text { DVP } \\
\text { (1 semana) }\end{array}$ & Total & Nenhuma & $\begin{array}{c}\text { RDT } \\
\text { neuroeixo }\end{array}$ & $6 \backslash 100$ \\
\hline 9 & $\begin{array}{c}\text { Craniectomia } \\
\text { suboccipital } \\
\text { bilateral }\end{array}$ & $\begin{array}{c}\text { Semi } \\
\text { sentada }\end{array}$ & $\begin{array}{c}\text { DVP } \\
(2 \text { dias })\end{array}$ & Subtotal & Nenhuma & RDT neuroeixo & $22 \backslash 100$ \\
\hline 10 & $\begin{array}{l}\text { Craniectomia } \\
\text { suboccipital } \\
\text { unilateral } \mathrm{E} \\
\text { hemilaminec- } \\
\text { tomia de } \mathrm{C} 1\end{array}$ & $\begin{array}{c}\text { Semi } \\
\text { sentada }\end{array}$ & DVE periop. & Subtotal & $\begin{array}{l}\text { Hematoma } \\
\text { leito cirúrgico } \\
\text { e ventriculite }\end{array}$ & $\begin{array}{c}\text { Encaminhada } \\
\text { a RDT }\end{array}$ & $\begin{array}{l}\text { sem seguimento, } \\
\text { alta com } 100\end{array}$ \\
\hline 11 & $\begin{array}{c}\text { Craniectomia } \\
\text { suboccipital } \\
\text { unilateral }\end{array}$ & $\begin{array}{c}\text { Semi } \\
\text { sentada }\end{array}$ & $\begin{array}{l}\text { DVE } \\
\text { periop. }\end{array}$ & Parcial & $\begin{array}{c}\text { Hematoma } \\
\text { leito cirúrgico } \\
\text { e ventriculite }\end{array}$ & $\begin{array}{c}\text { Não fez RDT, } \\
\text { aguarda reop. }\end{array}$ & $<1 \backslash 100$ \\
\hline 12 & $\begin{array}{l}\text { Craniectomia } \\
\text { suboccipital } \\
\text { unilateral E }\end{array}$ & Pronação & $\begin{array}{l}\text { DVE } \\
\text { periop. }\end{array}$ & Subtotal & $\begin{array}{c}\text { Hematoma } \\
\text { leito cirúrgico } \\
\text { e pneumotórax } \\
\text { intraop. }\end{array}$ & $\begin{array}{c}\text { reoperado e } \\
\text { efetuada } \\
\text { ressecção total, } \\
\text { encaminhado RDT }\end{array}$ & $<1 \backslash 100$ \\
\hline 13 & $\begin{array}{c}\text { Craniotomia } \\
\text { suboccipital } \\
\text { mediana }\end{array}$ & Pronação & $\begin{array}{l}\text { Cateter de } \\
\text { Lapras e } \\
\text { DVE periop. }\end{array}$ & Total & Nenhuma & $\begin{array}{c}\mathrm{RDT} \text { e QT, } \\
\text { reoperaçâo } \\
\text { após } 1^{\circ} \text { recidiva }\end{array}$ & $\begin{array}{c}31 \backslash 1^{\circ} \text { recidiva em } \\
\text { corno frontal } \\
\text { ventricular } \mathrm{D}(28 \\
\text { meses }) 100\end{array}$ \\
\hline 14 & $\begin{array}{c}\text { Craniotomia } \\
\text { suboccipital } \\
\text { mediana }\end{array}$ & Pronaçâo & $\begin{array}{c}\text { Cateter } \\
\text { de Lapras }\end{array}$ & Subtotal & Nenhuma & RDT & $55 \backslash 100$ \\
\hline 15 & $\begin{array}{r}\text { Craniectomia } \\
\text { suboccipital D }\end{array}$ & Pronação & $\begin{array}{l}\text { DVE } \\
\text { pré-op. }\end{array}$ & Total & Nenhuma & & \\
\hline
\end{tabular}

RDT, radioterapia; QT, quimioterapia; DVE, derivação ventricular externa; DVP, derivação ventrículo-peritoneal; D, direita; E, esquerda; periop, perioperatória; subarac, subaracnóideo; intraop,intra-operatório; reop, reoperação; pré-op, pré-operatório. 


\section{RESULTADOS}

\section{a) Idade e sexo}

Foram estudados 8 pacientes do sexo masculino e 7 do sexo feminino, 53\% para $47 \%$ respectivamente ou uma relação de 1,1/1,0. A idade média foi 23,0 anos, com variação de 13 a 46 anos.

\section{b) Dados relativos ao comportamento clínico}

Sintoma inicial - A cefaléia foi o sintoma inicial mais frequente ( $60 \%$ dos casos), seguida por incoordenação motora em $6,6 \%$, cefaléia e alteração de marcha em $6,6 \%$, vômitos em $6,6 \%$, visão dupla em 6,6\% e coma em 6,6\%, hipoacusia súbita em 6,6\%.

Exame neurológico - A síndrome de hipertensão intracraniana (HIC) foi observada em $86,6 \%$ dos casos, síndrome cerebelar global em 46,6\%, síndrome cerebelar apendicular em 20\%, síndrome cerebelar axial em 6,6\%, síndrome de nervos cranianos V, VII, VIII e IX (Caso10) em 6,6\% e alteração do estado de consciência em $6,6 \%$.

Intervalo entre sintoma inicial e diagnóstico - O intervalo médio do aparecimento dos sintomas até o diagnóstico foi 6,9 meses, variando de 1 a 15 .

\section{c) Dados relativos aos achados radiológicos}

Tipo de exame - Para o diagnóstico inicial a tomografia computadorizada (TC) de crânio associada à ressonância nuclear magnética (RNM) foi utilizada em 12 (80\%) casos e isoladamente em 3 .

Localização e tamanho do tumor - No hemisfério cerebelar em 7 (46,6\%)casos, sendo que um destes ocupava a cisterna do ângulo ponto-cerebelar (Caso 10), na região vermiana em 4 (26,6 $\%)$ e nas regiões vermiana e hemisférica em outros $4(26,6)$. Extensão supratentorial foi observada em 2 (13,3\%)casos. Em 53,3 \% dos casos havia invasão do quarto ventrículo total ou parcialmente. O diâmetro médio do tumor, calculado no seu maior eixo, foi de $4,5 \mathrm{~cm}$ variando de 3 a $6,5 \mathrm{~cm}$.

Aspecto tomográfico - Apresentavam-se isoatenuantes em 46,6\% e hiperatenuantes em 53,3\% e captaram contraste, com hiperatenuação, $80 \%$ dos casos. Calcificações foram encontradas em $13,3 \%$ e não se encontraram sinais de hemorragia intratumoral ou cistos nesta série. Sinais tomográficos de hidrocefalia foram encontrados em $93,3 \%$ dos casos.

\section{d) Dados relativos à cirurgia}

Tipos de acesso - Craniectomia suboccipital unilateral foi realizada em 8 (53,3\%) casos, craniectomia suboccipital mediana em 6 (40\%), e craniotomia suboccipital mediana em $1(6,6 \%)$. (Figs 1 e 2). A retirada do arco posterior de C1 foi realizada em 2 (13,3\%)casos.

Derivações liquóricas - A derivação ventricular externa (DVE) pré-operatória foi realizada em $11(73,3 \%)$ casos, a derivação ventrículo-peritoneal (DVP) pré-operatória em 1 (6,6\%), inserida 7 dias previamente à cirurgia da fossa posterior, a derivação do terceiro ventrículo com o espaço subaracnóideo - através de catéter ocupando o quarto ventrículo foi necessária em 3 (20\%) e derivação ventrículo-peritoneal definitiva, nos pacientes "shunt" dependentes após fechamento por 48 horas da DVE, foi realizada em 3 (20\%) (vide Tabela 2).

Posição do paciente - A posição semi-sentada foi a empregada em $11(73,3 \%)$ casos e o decúbito ventral com a cabeça rodada lateralmente em 4 (26,6\%).

Grau de ressecção - A ressecção foi considerada radical, quando ao "olho" do cirurgião esta lhe pareceu total, sendo confirmada por CT pós-operatória em 8 (53,3\%) pacientes (Fig 3); subtotal quando implantes tumorais aderidos a estruturas vasculoneurais (implante tumoral) foram deixados por cautela, totalizando em torno de 75 a 95\% de ressecção, o que foi verificado em 6 casos (40 \%). Houve 1 caso apenas de ressecção parcial (6,6\% dos casos).

e) Complicações e mortalidade intra e pós-operatórias

Os hematomas pós-operatórios intradurais em leito cirúrgico foram os mais frequentes e verificados em $5(33,3 \%)$ casos, sendo necessária reoperação e drenagem do hematoma em um 


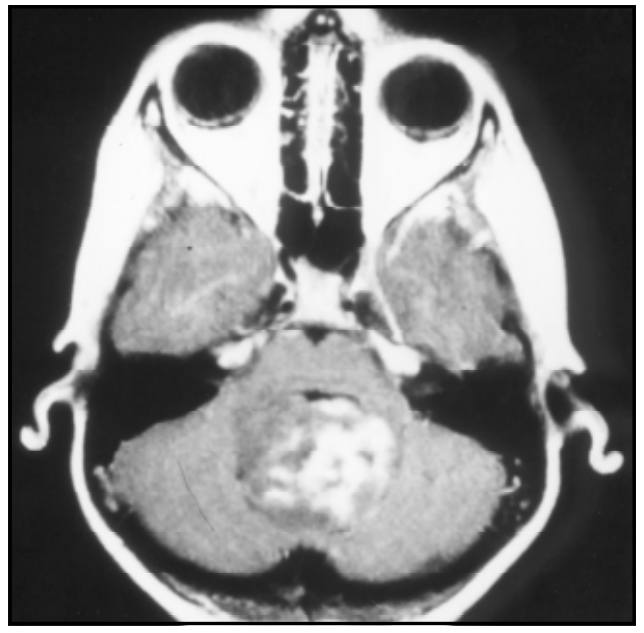

Fig 1. Caso 13. RNM de encéfalo, corte axial em T1 com gadolínio, mostrando invasão do quarto ventrículo, aderência ao tronco cerebral.

destes, indicação baseada na deterioração do nível de consciência e volume do hematoma. Observamos hematoma extradural em um paciente em que houve excesso de drenagem pela bolsa coletora da derivação ventricular externa $(6,6 \%)$ e que foi submetido à reoperação.

Os hematomas intradurais de leito cirúrgico foram mais frequentes nos pacientes:

1) submetidos a ressecção subtotal e parcial mais do que total (3 subtotais, 1 parcial, 1 total);

2) em tumores maiores que $5 \mathrm{~cm}$ de diâmetro (5 casos);

3) tumores vermianos e hemisféricos (3 casos) e hemisféricos (2 casos);

4) submetidos a craniectomia subocipital unilateral para tumores vermianos hemisféricos (2 casos), craniectomia suboccipital unilateral para tumor hemisférico (2 casos), craniectomia mediana para tumor vermiano hemisférico (1 caso).

Foram observados $2(13,3 \%)$ casos de ventriculite por Staphylococcus epidermidis, em pacientes submetidos a DVE perioperatória que foram tratados convenientemente com antibioticoterapia e manutenção prolongada da DVE. Houve lesão grave de nervos bulbares em um (6,6\%) caso e pneumotórax intra-operatório em outro $(6,6 \%)$.

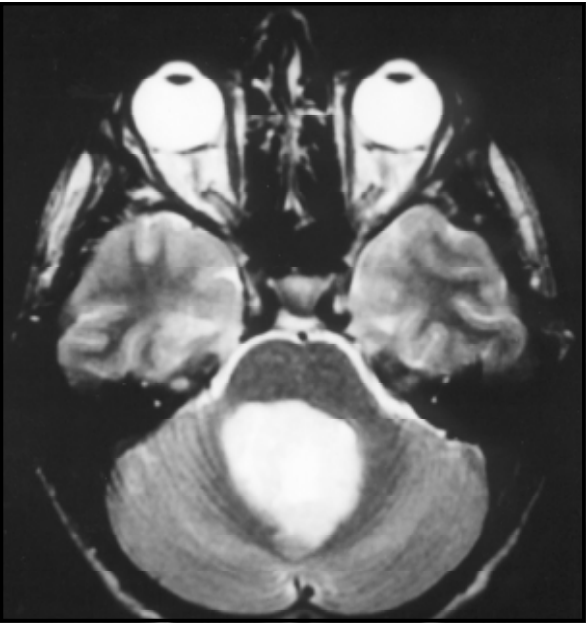

Fig 2. Caso 13. RNM de encéfalo, corte axial, aquisição em T2, mostrando lesão de hipersinal ocupando o quarto ventrículo

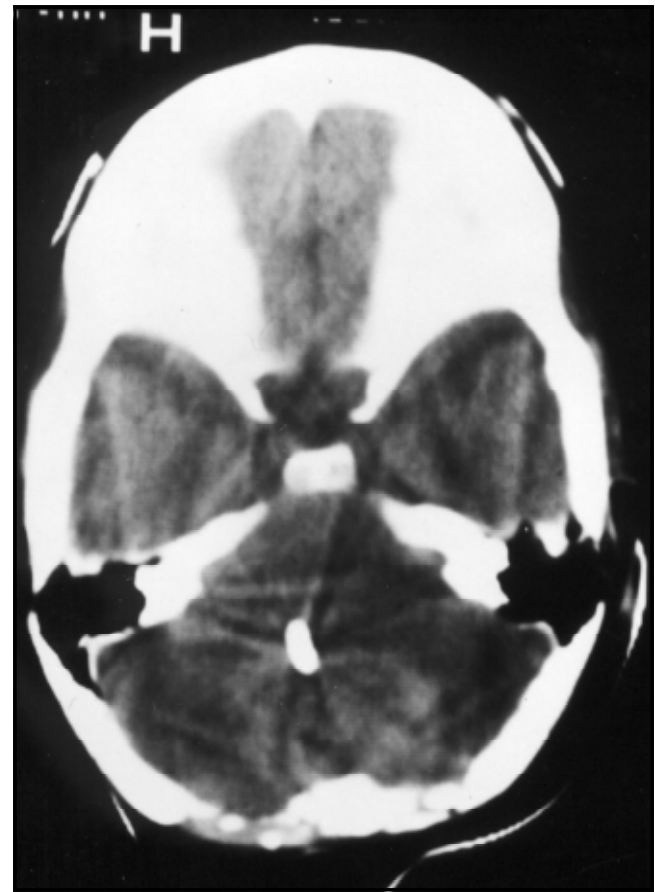

Fig 3. Caso 13. CT de crânio pós-opertória, corte axial, mostrando ressecção radical do tumor, cateter de Lapras em quarto ventrículo. 
Não houve complicação relativa ao posicionamento, como embolia gasosa, nos pacientes operados em posição semi-sentada.

Não houve mortalidade intra-operatória e no pós-operatório imediato, considerado até 7 dias.

\section{f) Segmento pós-operatório}

Tratamento adjuvante - 14 casos foram submetidos a radioterapia pós-operatória, 5400 rads em todo o neuroeixo. A quimioterapia foi empregada em 4 pacientes com recidiva e a droga utilizada foi o bromo-cloroetilnitrozuréia (BCNU).

Tempo de seguimento - Foi 22,7 meses (variando de um mês a 72 meses). Em apenas um caso não foi realizado seguimento.

Índice de Karnofsky por ocasião da última avaliação - 100\% em 12 (79,2\%) casos, o que é considerado bom resultado; $50 \%$ em um caso e $40 \%$ em 1 outro (maus resultados). Morbidade imediata de $40 \%$ (6 casos em 15 complicaram no período peri-operatório) e a morbidade tardia foi de $14,28 \%$ (2 casos com mau resultado tardio em 14 que foram seguidos).

Recidiva - 4 (28,5\%) casos apresentaram recidiva (4:14), e destes, 2 (50\%) apresentaram recidiva pela segunda vez (2:4). O tempo livre de doença para a primeira recidiva em média foi 29,2 meses (variando de 12 a 54 meses) e para a segunda recidiva foi 4,5 meses após a primeira recidiva. As recidivas se distribuíram do seguinte modo: 4 locais, 1 em corno frontal (em 1 paciente com catéter de Lapras, Caso13, Fig 3), 1 no hemisfério contralateral (Caso 2).

Reoperação - 3 casos foram reoperados na primeira recidiva e apenas um caso de primeira recidiva (Caso 2) foi submetido a quimioterapia, por falta de condições clínicas para a reoperação.

Sobrevida global - Segundo análise de curva actorial, a sobrevida global em 5 anos foi 93,3\%, com 11 casos sob suspeita (que não completaram a observação de 5 anos, mas que foram plotados na curva actorial), um caso com perda de seguimento que foi colocado na curva actorial, e $1(7,14 \%)$ caso de óbito, constituindo a mortalidade tardia (1 caso em 14 seguidos). Este caso, tratava-se de segunda recidiva local com infiltração de tronco cerebral (Caso 4). Não utilizamos a curva de Kaplan Meyer, nesta análise, em virtude do número pequeno de pacientes que foram observados em 5 anos. Os resultados encontram-se sumariados nas Tabelas 1 e 2.

\section{DISCUSSÃO}

Meduloblastoma é o modelo de tumor neuroectodérmico primitivo (PNET) infratentorial ${ }^{3,17}$. Desde 1925, Bailey e Cushing ${ }^{1}$ cunharam este termo para descrever um tumor maligno cerebelar ocorrendo principalmente em crianças, sendo um tumor vermiano densamente celular e distinto de sarcomas e outros gliomas. Estes autores imaginavam o meduloblasto como sendo uma célula neuroglial primitiva bipotente, capaz de se diferenciar em componente neuronal ou glial do sistema nervoso central (SNC). Esta entidade , apesar de apoiada por vários autores ${ }^{17}$, tem sido objeto de controvérsia, não se identificando o meduloblasto até o momento, persistindo dúvidas acerca da origem deste tumor.

Acredita-se, atualmente ${ }^{18}$, que um grupo de células neuroepiteliais, localizadas no teto do quarto ventrículo, migra em direção lateral e superficial de modo a formar a camada granular externa do cerebelo. Estas células persistem até a idade de um ano e acredita-se serem as responsáveis pelo surgimento dos meduloblastomas. Inferimos que podemos encontrar meduloblastomas em qualquer parte do trajeto destas células, o que poderia explicar porque pacientes mais idosos apresentam massas mais laterais, conforme encontramos tanto em séries de avaliação radiológica ${ }^{19}$ quanto patológica ${ }^{18}$.

Em oposição aos tumores supratentoriais, os meduloblastomas são tumores comuns, ficando em segundo lugar entre os tumores infantis de fossa posterior, sendo os astrocitomas pilocíticos os mais comuns, computando 13 a $28 \%$ dos tumores nesta faixa etária ${ }^{9,11}$. Mais de $50 \%$ dos $\operatorname{casos}^{11,15,18}$ ocorrem na primeira década principalmente entre os 5 e 10 anos de vida. $\mathrm{O}$ tumor é incomum na 
faixa adulta (acima de 16 anos), representando não mais do que 1\% dos tumores cerebrais nesta faixa etária (em nossa casuística a idade media foi de 23,0 anos, com variação entre 13 e 46 anos).

$\mathrm{Na}$ literatura existe predominância de pacientes masculinos, em proporção de $4: 3^{18,20}$. Não encontramos correspondência com a literatura, havendo apenas um leve predomínio de pacientes do sexo masculino em nossa série $(1,1: 1,0)$.

Os sintomas mais comuns são inespecíficos incluindo cefaléia, náusea e vômitos (86,6\% dos pacientes em nossa casuística com sinais e sintomas de hipertensão intra craniana) além de déficits visuais e motores, sendo a história clínica de curta duração (média de 6,9 meses em nossa série), de acordo com o comportamento de uma massa de crescimento rápido. O quarto ventrículo é frequentemente comprimido gerando hidrocefalia em 85 a $95 \%$ dos casos ${ }^{19}$. Seu comportamento é similar ao dos ependimomas, podendo estender-se para a cisterna magna via forame de Magendie e ângulo ponto-cerebelar via forame de Luschka ${ }^{3,19}$. A disseminação para o espaço subaracnóideo e ventrículos ocorre mais comumente que em outras lesões de fossa posterior ${ }^{18}$.

Estes tumores são geralmente vermianos com bordas delimitadas ${ }^{18}$ (constituindo 26,6\% em nossos pacientes, sendo $46,6 \%$ hemisféricos e $26,6 \%$ hemisféricos e vermianos). São friáveis e sangrantes com áreas de necrose central. Crescem para o quarto ventrículo (53,3\% em nossa série) e têm grande tendência a disseminação para o espaço subaracnóideo, especialmente como lesões na região lombar ou aderidas a raízes nervosas. Tumores laterais são vistos em adultos jovens e tendem a ser do tipo desmoplásico. Estes são tumores ricos em reticulina e bandas fibrosas com um corpo estromal, de consistência fibrosa à análise macroscópica.

Quando avaliamos nossa casuística, vemos que, do ponto de vista de tática cirúrgica o posicionamento do paciente em posição semi-sentada (73,3\%) foi o mais vantajoso, propiciando um grau de ressecção radical e subtotal em, respectivamente, 53,3 e 40\% dos casos tendo o auxílio de derivação ventricular externa em 73,3\% dos casos (46,6\% dos pacientes necessitando de derivações definitivas, interna ou ventrículo peritoneal).

A maioria dos pacientes $(93,3 \%)$ foi submetida a craniectomia, tendo como principal complicação o hematoma de leito cirúrgico $(33,3 \%)$, o que é compatível com a característica de tumor vascularizado como é o meduloblastoma, sendo que os hematomas,comumente,foram observados em casos submetidos a ressecções incompletas do processo.

Apesar do alto índice de complicações (40\% recentes e 14,28\% tardias), verificamos sobrevida em 5 (93,3\%) anos com Karnofsky de 100\% em 79,2\% dos casos.

Ao final da análise ressaltamos que, embora os meduloblastomas permaneçam sendo tumores de evolução desfavorável, situados em região sujeita a alto índice de complicações peri-operatórias, evoluímos muito em seu tratamento ${ }^{21,22}$, podendo fornecer aos nossos pacientes a esperança de uma sobrevida com qualidade.

\section{REFERÊNCIAS}

1. Bailey P, Cushing H. Medulloblastoma cerebelli: a common type of midcerebellar glioma of childhood. Arch Neurol Psychiatry 1925;14:192-224.

2. Choux M, Lena G, Hassoun J. Prognosis and long term follow-up in patients with medulloblastoma. Clin Neurosurg 1983;30:246-277.

3. Hart MN, Earle KM. Primitive neuroectodermal tumors of the brain in children. Cancer 1973;32:890-896.

4. Hazuka MB, DeBiose DA, Henderson RH, Kinzie JJ. Survival results in adult patients treated for medulloblastoma. Cancer 1992;69:2143-2148.

5. Arseni C., Ciurea AV. Statistical survey of 276 cases of medulloblastoma (1935-1978). Acta Neurochirurg 1981;57:159-162.

6. Bloom HJG, Bessel EM. Medulloblastoma in adults: a review of 34 patients treated between 1952 and 1981. Int J Radiat Oncol Biol Phys 1990;18:763-772.

7. Chatty EM, Earle KM. Medulloblastoma: a report of 201 cases with emphasis on the relationship of histologic variants to survival. Cancer 1971;28:977-983.

8. Cornu PH, Chatellier G, Fauchon F, et al.. Prognostic des médulloblastomas de l'adulte. Neurochirurgie 1990;36:218-224.

9. Ferrante L, Mastronardi L, Celli P, Acqui M, Cervoni L, Fortuna A.: Medulloblastoma in adulthood. J Neurosrg Sci 1991;35:23-30. 
10. Haie C, Schllenger M, Constans SP, Meder SF, Reynaud A, Ghenin C. Results of radiation treatment of medulloblastoma in adults. Int J Radiat Oncol Biol Phys 1985;11:2051-2056

11. Hubbard JL, Schitauer BW, Kispert DB, Carpenter SM, Wick MR, Laws ER. Adult cerebellar medulloblastomas: the pathological, radiographic, and clinical disease spectrum. J Neurosurg 1989;70:536-544.

12. Huges PG. Cerebellar medulloblastoma in adults. J Neurosurg 1984;60:994-997.

13. Lee BCP, Kneeland JB, Deck MDF. Posterior fossa lesions: magnetic resonance imaging. Radiology 1984;153:137-143.

14. Marty-Double C, Barneon G. Le médulloblastome de l'adulte (à propos d'une serie de 22 cas). Ann Anat Pathol 1974;60:994-997.

15. Pobereskin L, Treip C. Adult medulloblastoma. J Neurol Neurosurg Psychiatry 1986;49:39-42.

16. Dhellemmes P, Demaile MC, Lejeune JP, Baranzelli MC, Combelles G, Torrealba G. Cerebellar medulloblastoma: results of multidisciplinary treatment. Report of 120 cases. Surg Neurol 1986;25:290-294.

17. Rorke LB. The cerebellar medulloblastoma and its relationship to primitive neuroectodermal tumors. J Neuropathol Exp Neurol 1983;42:1-15.

18. Rubinstein LJ. Tumors of the central nervous system. Washington, DC: Armed Forces Institute of Pathology, Fasc. 6:104-158.

19. Zimmerman RA, Bilaniuk L, Pahlajani H. Spectrum of medulloblastomas demonstrated by computed tomography. Radiology 1978; 126:137-141.

20. Seitz WS, Opershall EJ. Medulloblastoma: example of late adult onset with statistical correlation relative to etiology. Radiol Clin 1978;47:395-409.

21. Belza MG, Donaldson SS, Steinberg GK, Cox RS, Cogen PH. Medulloblastoma: freedom from relapse longer than 8-years a therapeutic cure? J Neurosurg 1991;75:575-582.

22. Hartsel WF, Montag AG, Lydon J, Galinsky DL, Sarin P. Treatment of medulloblastoma in adults. Am J Clin Oncol 1992;15:207- 211. 